\title{
Prevalence of stroke in Restless Legs Syndrome: Initial Results Point to the Need for More Sophisticated Studies
}

\author{
Arthur S. Walters*, ${ }^{,}$, Michael Moussouttas ${ }^{2}$, Fouzia Siddiqui ${ }^{3}$, Diosely C. Silveira ${ }^{4}$, Karel Fuentes ${ }^{4}$, \\ Lily Wang ${ }^{5}$ and Klaus Berger ${ }^{6}$
}

${ }^{l}$ Dept of Neurology Vanderbilt University School of Medicine, Nashville, Tennessee, USA ${ }^{2}$ Dept of Neurology Thomas Jefferson Medical School, Philadelphia Pennsylvania, USA; ${ }^{3}$ Dept of Neurology, University of Toledo Medical Center, Toledo, Ohio USA; ${ }^{4}$ Dept. of Neurology, Cleveland Clinic, Cleveland, Ohio, USA; ${ }^{5}$ Dept of Biostatistics, Vanderbilt University School of Medicine; ${ }^{6}$ Institute of Epidemiology and Social Medicine, University of Muenster, Germany

\begin{abstract}
Background: Recent studies suggest a potential relationship between Restless Legs Syndrome (RLS) and hypertension and heart disease. Acute clinical stroke has been linked to the immediate onset of RLS, and epidemiological studies suggest the possibility that RLS may also lead to stroke.

Methods: MRI scans from 26 RLS cases and 241 controls from the population based MEMO-Study (Memory and Morbidity in Augsburg Elderly) were assessed for the presence of clinical stroke, silent infarction, subcortical lesions and cortical atrophy. T1, T2, proton density images were obtained and infarcts and their characteristics were determined by visual inspection. RLS status was assessed according to the minimal criteria of the International RLS Study Group. Scans from the 26 RLS patients and a subset of 26 age and sex matched controls were reexamined by a separate rater using the same methodology. Descriptive statistics, logistic and linear regression models were used to determine the risk of the three types of CNS changes associated with RLS case status.

Results: Among the 267 participants there was no difference in the prevalence of cardiovascular diseases or risk factors between RLS patients and the 241 controls. The prevalences of cerebrovascular events of all types, were greater in RLS patients, as were the amounts of cortical atrophy and the volume of subcortical lesions. However, these differences were not statistically significant. When age, sex and co-morbidities were taken into account in a logistic regression model, there was a statistically non-significant greater risk for stroke (Odds Ratio 2.46 with 95\% CI 0.97-6.28, p = .06) associated with RLS case status.

Conclusions: Future similar studies need to be performed on younger patients without other potential vascular risk factors, using Flair images and computerized programs for detection of cerebral ischemia. Improved methods for detection may allow for a reasonable sample size.
\end{abstract}

Keywords: Magnetic resonance imaging, restless legs syndrome, cerebrovascular disease, stroke, cardiovascular disease, hypertension, periodic limb movements in sleep, leukoaraiosis.

\section{INTRODUCTION}

Recent evidence has been accumulating that heart disease, hypertension and stroke are linked to Restless Legs Syndrome/Periodic Limb Movements in Sleep (RLS/PLMS [1]. Although the links between RLS and heart disease have been quite consistent, an association of RLS with hypertension is reported in some epidemiological studies [2-4] but not in others [5-8]. On the other hand, direct measurement of the numbers of PLMS and the level of daytime hypertension simultaneously suggest that there is a direct correlation [9, 10]. Theoretical mechanisms as to how RLS/PLMS might be linked to heart disease and stroke in the absence of hypertension are discussed elsewhere [1]. In three initial independent case reports and one larger later case series of 17 of 137 stroke patients, RLS or its accompanying PLMS, began

*Address correspondence to these authors at the Dept of Neurology, Vanderbilt University Medical Center, Medical Center North Room A-0118, $116121^{\text {st }}$ Ave South, Nashville, Tennessee 37232-2551, USA; Tel: 615322-0283; Fax: 615-936-0223;

E-mail: artumdnj@aol.com, arthur.walters@ vanderbilt.edu immediately after acute clinically evident stroke primarily in the area of the basal ganglia, corona radiata or other subcortical areas [11-14]. Preliminary results from a small Magnetic Resonance Imaging (MRI) study suggest that RLS is also associated with silent infarcts [15]. Thus, although stroke may lead to RLS/PLMS, the possibility remains that RLS/PLMS may also lead to stroke. This possibility was explored in an epidemiologic study by Elwood et al. where 1986 men aged 55-69 years completed a questionnaire regarding RLS and other sleep disorders. During the following 10 years 107 men experienced an ischemic stroke and 213 had an ischemic heart event. Compared with men who reported no symptoms of sleep disturbance of any kind, the relative odds of an ischemic stroke was 1.67 (95\% CI: 1.07$2.60-\mathrm{P}=.024)$ in patients who responded positively to a question on RLS [16].

The aim of our study was to examine the relationship between RLS and silent as well as clinically evident stroke and subcortical lesions on MRI in a population based study of the elderly. 


\section{METHODS}

The MEMO (Memory and Morbidity in Augsburg Elderly) Study [8] is a follow-up project of the 1989-90 World Health Organization "Monitoring Trends and Determinants in Cardiovascular Disease" (MONICA) Survey in Augsburg, Southern Germany. MEMO examines cognitive function and risk factors for neurodegenerative diseases in the elderly. It was part of the "Cardiovascular Determinants of Dementia (CASCADE) Study" a European multicenter study with the objective of evaluating the effects of cardiovascular risk factors on cerebral morphology among community-dwelling elderly men and women. The MEMO Study was restricted to participants of the second MONICA survey, who were aged 65 or older on October 1, 1997, and lived in the city of Augsburg or one of two neighboring suburbs. Sampling of participants was done by age group $(65-69,70-74,>75)$ to represent a broad range between age 65 and 83 . The overall response rate of the eligible $(n=635)$ MONICA participants was $60.6 \%$, yielding 385 MEMO participants. Informed consent was obtained from all study participants before their examination.

\section{MRI Measurements}

A cerebral MRI of the brain was performed in 267 study participants without contraindications. 118 participants did not receive an MRI because of metal body implants, artificial eye lenses or claustrophobia, or refusal of scanning. Participants with MRI were slightly younger (72.3 versus 73.7 years, $\mathrm{p}=0.003)$ and more often male (52.2 versus $47.8 \%$, $\mathrm{p}=0.77)$. All MRI scans were performed using a Phillips (Best, The Netherlands) 1.5 tesla machine at the Department of Radiology, Central Hospital of Augsburg. The MRI protocol included proton density (PD), T1, and T2 weighted images acquired with spin echo sequences with 20 axial slices, 5 or $6 \mathrm{~mm}$ thick, with an interslice gap of 1 or 1.2 $\mathrm{mm}$, respectively. All MRI scans were first examined visually by a single reader for periventricular and subcortical lesions, infarcts, and cortical as well as subcortical atrophy using an established rating scale [17]. Subcortical lesions were focal signal hyperintensities on proton density and T2weighted scans, which were not seen or exhibited only faint hypointensity on T1-weighted images. Periventricular lesions were abnormalities directly abutting the lateral ventricle. They were graded on a severity scale (0-3) at the frontal and occipital horns and the body of the lateral ventricle, with the total periventricular lesion score being the sum of these three scores. Subcortical lesions were hyperintense foci separated from the lateral ventricle. They were graded by size (small, medium, and large) and number. The total volume of subcortical lesions was assessed by multiplying each lesion by a size-dependent constant $(0.0042$ for small lesions, 0.114 for medium lesions, and 0.95 for large lesions) and by subsequent summation of the results. Infarcts were focal abnormalities with clearly defined borders, and they were isointense to cerebrospinal fluid on all sequences with a diameter $>2 \mathrm{~mm}$. Cortical atrophy was qualitatively assessed by a semi quantitative scale (0-3) applied to each lobe and to the sylvian fissure. The sum of the lobar scores represented the total cortical atrophy score (range 0-15). Subcortical atrophy was defined as the average of ventricular indexes relative to the width of the hemispheres measured at the level of frontal horns, occipital horns, and caudate nuclei [18].
The MRI scans of 26 RLS cases and 26 of the 241 noncases matched for age and sex, were examined visually a second time in a blinded fashion by one of us (MM) at Thomas Jefferson University in Philadelphia, PA, USA. This was implemented to determine the consistency of infarct quantification via the use of a different rating scale.(18) For the second rating MRI T2 and proton density images were used to quantify the degree of subcortical lesions (leukoaraiosis) using the scale developed by the European Task Force on Age Related Subcortical Changes, and described elsewhere [19]. Briefly, subcortical ischemic changes were considered ill-defined hyperintensities $>=5 \mathrm{~mm}$ in diameter, and lacunar infarctions were considered well-defined areas of $>2 \mathrm{~mm}$ diameter with characteristics similar to CSF. Five different areas were assessed bilaterally for the presence of such lesions - the frontal areas, the parieto-occipital areas, the temporal areas, the diencephalic region (including the striatum, pallidum, thalamus, internal/external capsules, and insula), and the infratentorial region (including pons, cerebellum, mesencephalon, and medulla). Within the diencephalic region, lesions were scored as 0 if none were present that met measurement criteria, as 1 if a single focal lesion was present, as 2 if more than one lesion was present, and as 3 if lesions were confluent. Within the remaining anatomic locations, leukoaraiosis was scored as 0 if no lesions were present that met measurement criteria, as 1 if only focal lesions were present, as 2 if the lesions were confluent, and as 3 for diffuse involvement. A maximum total of 30 points per scan/patient were possible based on this rating scale. The correlation between these two classifications based on different scales was high (spearman rank correlation coefficient $\mathrm{rho}=0.78, \mathrm{p}<0.001)$.

\section{Other Methodology}

General medical history, history of neurological and psychiatric diseases, family history, and current medication was assessed in a face to face interview in all participants. A stroke classification based on clinical symptoms was defined as one or more positive answers to a stroke symptom questionnaire and subsequent validation by medical records [20]. Silent infarction was defined as the presence of a hypodense (T1) or hyperdense (T2, proton density) lesion of $>2 \mathrm{~mm}$ on the MRI scan and the answer 'No' to the question 'Did you ever have a stroke that was diagnosed by a physician?' made by the respective participant. In addition, all participants received a standardized neurological examination by one of two physicians. RLS was diagnosed according to questions assessing the International RLS Study Group diagnostic criteria $[8,21]$. The RLS diagnosis was validated by either of the two physicians who were blinded to the results of the questions. In the group of MEMO participants with MRI this yielded twenty six RLS cases and 241 non cases. Blood was collected in all participants in a standardized way directly after the interview. The intima media thickness of the carotid artery was determined by B-mode ultrasonography using a standard protocol, but restricted to 120 participants due to logistic reasons.

\section{Statistical Analysis}

Differences in categorical variables were compared between RLS cases and non-cases using the chi-squared test or the Fisher's exact test (if more than $20 \%$ of the expected 
counts were 5 or less). Differences in continuous variables were tested with Student's t-test. Serum levels for CRP and the subcortical lesion volume variable were log-transformed to approximate normal distribution. A logistic regression model was applied to determine the Odds Ratio (OR) of RLS case status for the dichotomized (yes-no) variables silent infarction, all stroke, and presence of large subcortical lesions. These outcomes were the dependent variable in the respective model and RLS case, age, gender and co morbidities were included as the independent variables. For the subcortical lesion volume and the cerebral atrophy analysis a linear regression model was used with the log-transformed volume and the atrophy score being the respective dependent variable and the variables listed above being the independent variables. All analyses were done using the Stata statistical package (version 9.0).

For the comorbidity adjustment two grouping variables were generated, one included vascular comorbidities (histories of either myocardial infarction or diabetes or heart failure or operation of the heart or large arteries), the other joint and bone related conditions (histories of either arthritis or operation of the large joints). These two grouping variables were coded 1 if one or more of the listed comorbidities were reported. Grouping of comorbidities was done to restrict the number of adjustment variables given the limited number of RLS cases.

\section{RESULTS}

The prevalence of RLS among the 267 study participants with a cerebral MRI was $9.7 \%$. This is comparable to the prevalence of RLS found in population based studies [2-7]. The overall prevalence of silent infarction in this sample was $12.7 \%$ and that of all strokes, regardless if detected by MRI or clinical history or both, was $19.5 \%$.

There was no significant difference in the prevalence of vascular risk factors or medical conditions between the 26 RLS patients and all 241 non-cases (Table 1). There was a clearly higher all-stroke prevalence among the RLS cases than among non-cases, but this difference was not statistically significant (Table 2). When age, sex and co- morbidities were taken into account in a logistic regression model, RLS case status was associated with a higher non-significant risk for stroke (Odds Ratio 2.46 with 95\% CI 0.97-6.28, $\mathrm{p}=0.06$ ). The prevalence of silent infarctions and large subcortical lesions was also higher among the 26 RLS cases compared to the 241 controls but this result was also not statistically significant (Table 2). Similarly, the RLS cases had slightly more affected subcortical (volume) and more cerebral atrophy than the controls in univariate analysis. These differences were also not statistically significant. Additional adjustment for age, sex and comorbidities in a linear regression model with cerebral atrophy score as the dependent variable did not change the conclusions of these results either. Sensitivities, specificities, positive and negative pre-

Table 1. Clinical Characteristics of the MEMO Study Participants According to RLS Status

\begin{tabular}{|c|c|c|c|}
\hline & $\begin{array}{l}\text { RLS Cases } \\
\qquad \mathbf{N}=\mathbf{2 6}\end{array}$ & $\begin{array}{l}\text { Controls } \\
\mathrm{N}=\mathbf{2 4 1}\end{array}$ & $\mathbf{P}$ \\
\hline Age, mean & $71.8(4.2)$ & $72.3(4.4)$ & 0.62 \\
\hline Diastolic. Blood pressure, mean & $81.7(7.3)$ & $82.2(9.4)$ & 0.78 \\
\hline Hypertension measured ${ }^{1}, \%$ & 61.5 & 60.2 & 0.89 \\
\hline History ${ }^{2}$ of diabetes, $\%$ & 0.0 & 9.1 & 0.25 \\
\hline Blood glucose, mean mg/dl & $96.5(2.2)$ & $106.9(2.4)$ & 0.14 \\
\hline History $^{2}$ of myocardial infarction, $\%$ & 3.9 & 7.5 & 0.79 \\
\hline History $^{2}$ of heart failure, $\%$ & 7.7 & 10.0 & 0.18 \\
\hline HDL-Cholesterol, mean mg/dl & $50.3(9.7)$ & $49.7(13.2)$ & 0.82 \\
\hline $\mathrm{Ln} \mathrm{Lpa}^{3}$, mean $(\mathrm{SD}) \mathrm{mg} / \mathrm{dl}$ & $3.22(1.06)$ & $3.21(1.15)$ & 0.65 \\
\hline $\mathrm{Ln} \mathrm{CrP}^{4}$, mean $(\mathrm{SD}) \mathrm{mg} / \mathrm{dl}$ & $0.53(1.10)$ & $0.70(1.07)$ & 0.45 \\
\hline Creatinine, $\mathrm{mg} / \mathrm{dl}$ & $1.13(0.49)$ & $1.08(0.30)$ & 0.48 \\
\hline $\begin{array}{l}\text { Intima media right, mean mm } \\
\text { Thickness }{ }^{5} \text { left, mean mm }\end{array}$ & $\begin{array}{l}0.887(0.127) \\
0.909(0.108)\end{array}$ & $\begin{array}{l}0.916(0.118) \\
0.918(0.124)\end{array}$ & $\begin{array}{l}0.41 \\
0.78\end{array}$ \\
\hline
\end{tabular}

Numbers in parentheses indicate standard deviations.

${ }^{1}$ Systolic blood pressure $>=140 \mathrm{mmHg}$ and $/$ or diastolic blood pressure $>=90 \mathrm{mmHg}$.

${ }^{2}$ Self reported history of a doctor diagnosis.

${ }^{3}$ Means (SD) of log-transformed Lpa values, representing geometric means of (cases) and (non-cases).

${ }^{4}$ Means (SD) of log-transformed CrP values, representing geometric means of (cases) and (non-cases).

${ }^{5}$ Restricted to 14 cases and 106 controls. 
Table 2. MRI Findings According to RLS Case Status

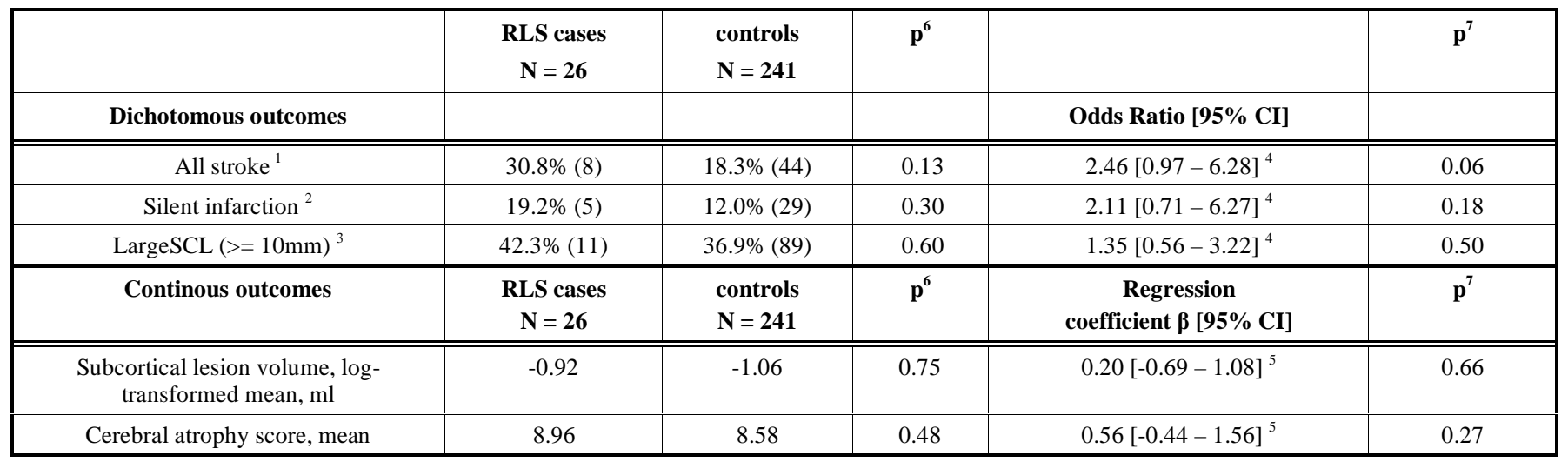

For Dichotomous outcomes numbers in parentheses equal number of patients.

${ }^{1}$ Detected by MRI and/or by clinical history validated through medical record review.

${ }^{2}$ Defined as an answer "no" to the question about physician diagnosed stroke but with an ischemic lesion on MRI.

${ }^{3}$ SCL denotes large subcortical lesion.

${ }^{4}$ Odds ratio and its $95 \%$ confidence interval, derived from logistic regression analysis with RLS status, age, gender and co morbidities as independent variables.

${ }^{5}$ Regression coefficient and its $95 \%$ confidence interval, derived from linear regression analysis with RLS status, age, gender and co morbidities as independent variables.

${ }^{6}$ Unvariate p-value.

${ }^{7} \mathrm{P}$-value corresponding to the confidence interval.

dictive values for the relationship of stroke to RLS are shown in Table 3.

\section{DISCUSSION}

The major finding of this study is that all determined cerebrovascular changes were more frequently observed among RLS cases compared to non-cases. However, these differences did not reach statistical significance. The small number of RLS patients is one likely explanation of the lack of statistical significance between RLS and silent infarcts. Indeed a power analysis revealed that this study had a power of $27 \%$ and $15 \%$ to detect a significant difference between cases and non-cases for all strokes and silent infarcts, respectively, given the observed prevalences of both. This would imply that a very large sample size would be needed for future studies if the same methods would be applied. However, application of newer MRI techniques such as Flair images that were not available in the MEMO Study, is likely to improve detection of ischemia and may help elucidate true differences between RLS cases and non-cases. In addition, application of sophisticated computer programs for MRI image analysis and infarct detection and measurement, as opposed to the visual rating of strokes by eye in the MEMO Study, might be helpful in this regard. Both the use of Flair images and computerized digital imaging analysis can contribute to enhance precision, sensitivity and, subsequently, might help to reduce sample sizes. Finally, recruiting patients and controls without other vascular risk factors may be important in detecting differences on MRI that could be attributed to RLS alone. This would also be expected to reduce sampler size. Indeed we did a power calculation based on a much smaller but similar study we performed, where Flair images were utilized, automated quantification of infarct burden was employed and, for the purposes of the power calculation, patients with hypertension were eliminated (15). The power analysis was based upon volume load of microvascular disease and not the presence or absence of lesions. A t value of 1.74 was obtained, corresponding to a standardized effect size (Cohen's d) of approximately .78. Assuming this value is clinically meaningful in the power analysis, it was estimated that power could be expected to reach an acceptable level of .80 if about 27 RLS patients and 27 controls were recruited [15].

Of course, an alternative explanation for our results is that they represent true negative findings. It is also conceivable that the patients more severely affected by cardiovascular and cerebrovascular disease did not volunteer or were unable to participate in this study.

Table 3. Sensitivity, Specificity, Positive and Negative Predictive Values and their 95\% Confidence Intervals for RLS as a Marker for Stroke

\begin{tabular}{|c|c|c|c|c|c|c|c|c|c|c|}
\hline & \multicolumn{3}{|c|}{ RLS Cases } & \multicolumn{3}{|c|}{ Controls } & \multirow[b]{2}{*}{ Sensitivity (CI) } & \multirow[b]{2}{*}{ Specificity(CI) } & \multirow[b]{2}{*}{ Pos Pred Value (CI) } & \multirow[b]{2}{*}{ Neg Pred Value (CI) } \\
\hline & Total\#RLS & Stroke & \#No Stroke & Total\#CTL & Stroke & \#No Stroke & & & & \\
\hline All Stroke & 26 & 8 & 18 & 241 & 44 & 197 & $0.15(0.06,0.25)$ & $0.92(0.88,0.95)$ & $0.31(0.13,0.49)$ & $0.82(0.77,0.87)$ \\
\hline Silent Stroke & 26 & 5 & 21 & 241 & 29 & 212 & $0.15(0.03,0.27)$ & $0.91(0.87,0.95)$ & $0.19(0.04,0.34)$ & $0.88(0.84,0.92)$ \\
\hline Large SCL & 26 & 11 & 15 & 241 & 89 & 152 & $0.11(0.05,0.17)$ & $0.91(0.87,0.95)$ & $0.42(0.23,0.61)$ & $0.63(0.57,0.69)$ \\
\hline
\end{tabular}

sensitivity = number of pts with RLS and stroke / total number of pts with stroke.

specificity $=$ number of pts without RLS and without stroke / total number of pts without stroke.

pos predictive value = \# of pts with RLS and stroke / \# of pts with RLS

negative predictive value $=\#$ of pts without stroke and without RLS / \# of pts without RLS

$\mathrm{CI}=95 \%$ confidence interval for the proportion.

$\mathrm{SCL}=$ Subcortical Lesion . 
A strength of this study is its population base, the possibility to perform MRI with a standardized protocol and that MRI scans were obtained at the time of evaluation for RLS. A limitation is that patients could only be evaluated for RLS on one occasion and that the severity of RLS was not assessed in order to associate the level of RLS severity with the number of silent infarcts on MRI scan. Another limitation is that this is a cross-sectional study and we do not have good information on what came first: the silent infarct or the RLS.

Theoretical mechanisms as to how RLS/PLMS could lead to stroke or stroke could lead to RLS/PLMS are discussed elsewhere in detail and are beyond the scope of the current publication [1].

\section{FUTURE DIRECTIONS}

Determining whether there is a connection between stroke and RLS is of no small importance from a clinical point of view. The potential mechanisms interlinking stroke and RLS are multiple and have been explored in detail elsewhere [1]. A key issue is whether or not the link between RLS and stroke is mediated by hypertension or not [1-8] and future studies should explore whether RLS is an independent risk factor for stroke or whether the risk for stroke in RLS is mediated through hypertension and other risk factors.

\section{CONCLUSIONS}

Based on recent publications reporting that patients with RLS and PLMS are at increased risk of hypertension, heart disease, and stroke, ${ }^{1}$ we analyzed the association between RLS and MRI determined vascular brain changes in the MEMO-Study. Using the study's cross-sectional design we only found statistically non-significant evidence for this association despite considerable raw absolute differences. Future studies should use prospective recruitment of patients and controls without other risk factors for stroke and the application of newer MRI sequences and automated image analyses for stroke detection.

\section{ABBREVIATIONS}

$$
\begin{array}{ll}
\text { MRI } & =\text { Magnetic Resonance Imaging } \\
\text { RLS } & =\text { Restless Legs Syndrome } \\
\text { PLMS } & =\text { Periodic Limb Movements in Sleep }
\end{array}
$$

\section{DISCLOSURE}

This is not an industry sponsored study. The authors report no conflicts of interest.

\section{ACKNOWLEDGEMENT}

The MEMO-Study is supported by the German Research Society (Deutsche Forschungsgemeinschaft, grant: BE1996/1-1). Data assessment was done within the framework of the Cooperative Health Research in the Augsburg Region (KORA). We would also like to acknowledge the statistical support of Barry Cohen, PhD of New York University, USA.

\section{REFERENCES}

[1] Walters AS and Rye DB. Review of the relationship of Restless Legs Syndrome / Periodic Limb Movements in Sleep to Hypertension. Heart Dis Stroke Sleep 2009; 32: 589-597.

[2] Ulfberg J, Nystrom B, Carter N, Edling C. Prevalence of Restless Legs Syndrome among men aged 18 to 64 years: An association with somatic disease and neuropsychiatric symptoms. Mov Disord 2001; 16: 1159-1163.

[3] Ohayon MM and Roth T. Prevalence of Restless Legs Syndrome and Periodic Limb Movement Disorder in the general population. J Psychosom Res 2002; 53: 547-554.

[4] Phillips B, Hening W, Britz P, Mannino D. Prevalence and correlates of Restless Legs Syndrome. Chest 2005; 129: 76-80.

[5] Winkelman JW, Shahar E, Sharief I, Gottlieb DJ. Associations of Restless Legs Syndrome and Cardiovascular Disease in the Sleep Heart Health Study. Neurology 2008; 70: 35-42.

[6] Winkelman JW, Finn L, Young T. Prevalence and correlates of Restless Legs syndrome symptoms in the Wisconsin Sleep Cohort. Sleep Med 2006; 7: 545-552.

[7] Hogl B, Kiechl S, Willeit J, et al. Restless Legs Syndrome: A community-based study of prevalence, severity, and risk factors. Neurology 2005; 64: 1920-1924.

[8] Rothdach AJ, Trenkwalder C, Haber Stock J, Keil U, Berger K. Prevalence and risk factors of RLS in an elderly population. Neurology 2000; 54: 1064-1068.

[9] Espinar-Sierra J, Vela-Bueno A, Luque-Otero M. Periodic Leg Movements in Essential Hypertension. Psych Clin Neurosci 1997; 51: $103-107$.

[10] Billars L, Hicks A, Bliwise D, Sigmundsson T, Sigurdsson A Kristjansson K, Gulcher J, Stefansson K, Rye D. Hypertension risk and PLMS in Restless Legs Syndrome. Sleep 2007; 30: A297A298.

[11] Kang SY, Sohn YH, Lee Il K, Kim J-S. Unilateral periodic limb movement in sleep after supratentorial cerebral infarction. Parkinson Rel Disord 2004; 10: 429-431'

[12] Lee JS, Lee PH, Huh K. Periodic limb movements in sleep after a small deep subcortical infarct. Mov Disord 2005; 20: 260-1.

[13] Anderson KN, Bhatia KP, Losseff NA. A case of restless legs syndrome in association with stroke. Sleep 2005; 28: 147-8.

[14] Lee SJ, Kim JS, Song IU, An JY, Kim YI, Lee KS. Poststroke restless legs syndrome and lesion location: anatomical considerations. Mov Disord 2009; 24 : 77-84.

[15] Silveira DC, Siddiqui F, Strus J, Fuentes K, Moussouttas M, Walters AS. Restless legs syndrome and cerebral microvascular ischemic disease. Neurology 2007; 68 (Suppl 1): A257.

[16] Elwood P, Hack M, Pickering J, Hughes, J, Gallacher J. Sleep disturbance, stroke, and heart disease events: evidence from the Caerphilly cohort. J Epidemiol Commun Health 2006; 60: 69-73.

[17] Schmidt W-P, Roesler A, Kretzschmar K, Ludwig K-H, Junker R, Berger K. Functional and cognitive consequences of silent stroke discovered using brain magnetic resonance imaging in an elderly population. J Am Geriatr Soc 2004; 52: 1045-1050.

[18] Schmidt R, Launer LJ, Nilsson L-G, et al. Magnetic resonance imaging of the brain in diabetes: The cardiovascular determinants of dementia (CASCADE) study. Diabetes 2004; 53: 687-692.

[19] Wahlund L, Barkhof F, Fazekas F, et al. A New Rating Scale for Age-Related White-Matter Changes Applicable to MRI and CT. Stroke 2001; 32: 1318-22.

[20] Berger K, Hense HW, Rothdach A, Weltermann B, Keil U. A single question about prior stroke versus a stroke questionnaire to assess stroke prevalence in populations. Neuroepidemiology 2000; 19: 245-57.

[21] Allen RP, Picchietti D, Hening WA, Trenkwalder C, Walters AS, Montplaisir J. The participants in the Restless Legs Syndrome Diagnosis and Epidemiology workshop at the National Institutes of Health in collaboration with members of the International Restless Legs Syndrome Study Group. Restless Legs Syndrome: diagnostic criteria, special considerations, and epidemiology. A report from the Restless Legs Syndrome diagnosis and epidemiology workshop at the National Institutes of Health. Sleep Medicine 2003; 4: 101119. 